\title{
Proyección del consumo de energía residencial en el Perú (2005-2030) mediante el software MAED D
}

\begin{abstract}
RESUMEN
El presente artículo muestra una metodología para determinar la proyección del consumo nacional de energía del sector residencial para el periodo 2005 2030 (Modelo para el análisis de demanda de Energía MAED_D).

Se empieza con una descripción de la evolución del sector energético nacional (oferta y demanda de fuentes primarias y secundarias), continúa con el análisis de las causas y efectos de la crisis energética, y de las variables explicativas del consumo de energía en el sector residencial. Se procede con la descripción del MAED_D y se usa el software ingresando los factores demográficos y los hábitos de consumo del sector residencial nacional. Los resultados obtenidos muestran que el consumo de energía tomando como base el año 2005 (4.536 GWa), se podría incrementar hasta 7.845 GWa en el año 2030.
\end{abstract}

Palabras clave: Proyección del consumo de energía residencial.

Projection of Residential Energy

Consumption in Peru (2005-2030)

\section{ABSTRACT}

This paper presents a methodology for determining the projection of national energy consumption in the household sector for the period 2005 - 2030 (Model for Energy Demand Analysis MAED_D).

The paper starts with the development of national energy sector (supply and demand of primary and secondary), continuing with the analysis of the main indicators of the energy crisis of the explanatory variables and energy consumption in the household sector. After this analysis is done using the software entered demographic factors and consumer habits of the household sector. The results show that energy consumption based on the year 2005 (4.536 GWa) could increase to 7845 GWa in 2030.

KEYWORDS: Household future energy consumption

\section{INTRODUCCIÓN}

La demanda nacional de energía en las últimas décadas se viene incrementado en forma exponencial, influenciado principalmente por el crecimiento económico y demográfico, las decisiones políticas, el desarrollo tecnológico y los hábitos de consumo o estilos de vida. Frente a estos acontecimientos, el gobierno no ha tenido una política energética concertada en el que la sociedad representativa establezca un plan energético nacional que permita el desarrollo del sector energético a mediano y largo plazo. El centro de planeamiento estratégico nacional, encargado de coordinar los esfuerzos de las diferentes entidades del Estado, no ha elaborado un plan energético nacional de energía, por lo que las políticas del gobierno de turno responden a situaciones de corto plazo.

El déficit de la balanza comercial de hidrocarburos y la dependencia de los precios internacionales del petróleo hacen que el precio de los combustibles sea elevado, lo que afecta en mayor medida a la población de más bajos recursos económicos.

Es necesario realizar evaluaciones proyectadas en el mediano y largo plazo de la demanda nacional de energía, los resultados servirán para la elaboración de un planeamiento energético integrado y concertado que permitirá tener señales oportunas para definir políticas energéticas generales, metas y plazos para asegurar el suministro de energía y el bienestar social, satisfacer la demanda y permitir el desarrollo sostenido utilizando recursos energéticos propios disponibles en cantidades suficientes a precios razonables y la inclusión progresiva de las energías renovables.

El modelo MAED_D es adecuado para proyectar la demanda nacional de energía porque evalúa la demanda futura utilizando escenarios de desarrollo socioeconómico (crecimiento demográfico y económico, hábitos de consumo y el desarrollo tecnológico), es más exacto que los modelos macroeconómicos que sólo consideran el PBI (en la conformación del PBI no se considera el uso de combustibles energéticos no comerciales: bosta, leña, yareta y bagazo, etc.).

\footnotetext{
Ingeniero Industrial. Profesor del Departamento de Diseño y Tecnología Industrial UNMSM, E-mail: orojasl@unmsm.edu.pe

2 Ingeniero Mecánico, egresado de la maestría en Ciencias con mención en energética de la UNI, E-mail: jorger2@hotmail.com
} 
FIGURA 1. CONSUMO NACIONAL DE ENERGÍA FINAL POR SECTORES (2007)

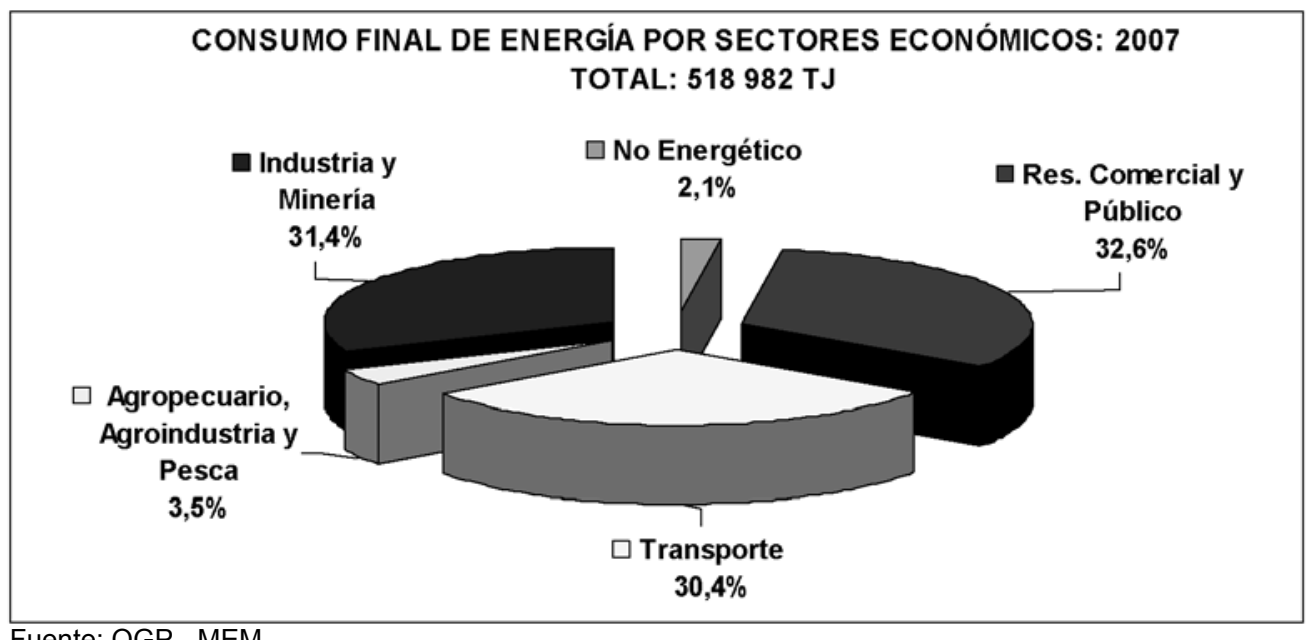

Fuente: OGP - MEM.

FIGURA 2. EVOLUCIÓN DE LA OFERTA DE ENERGÍA PRIMARIA (1998-2007)

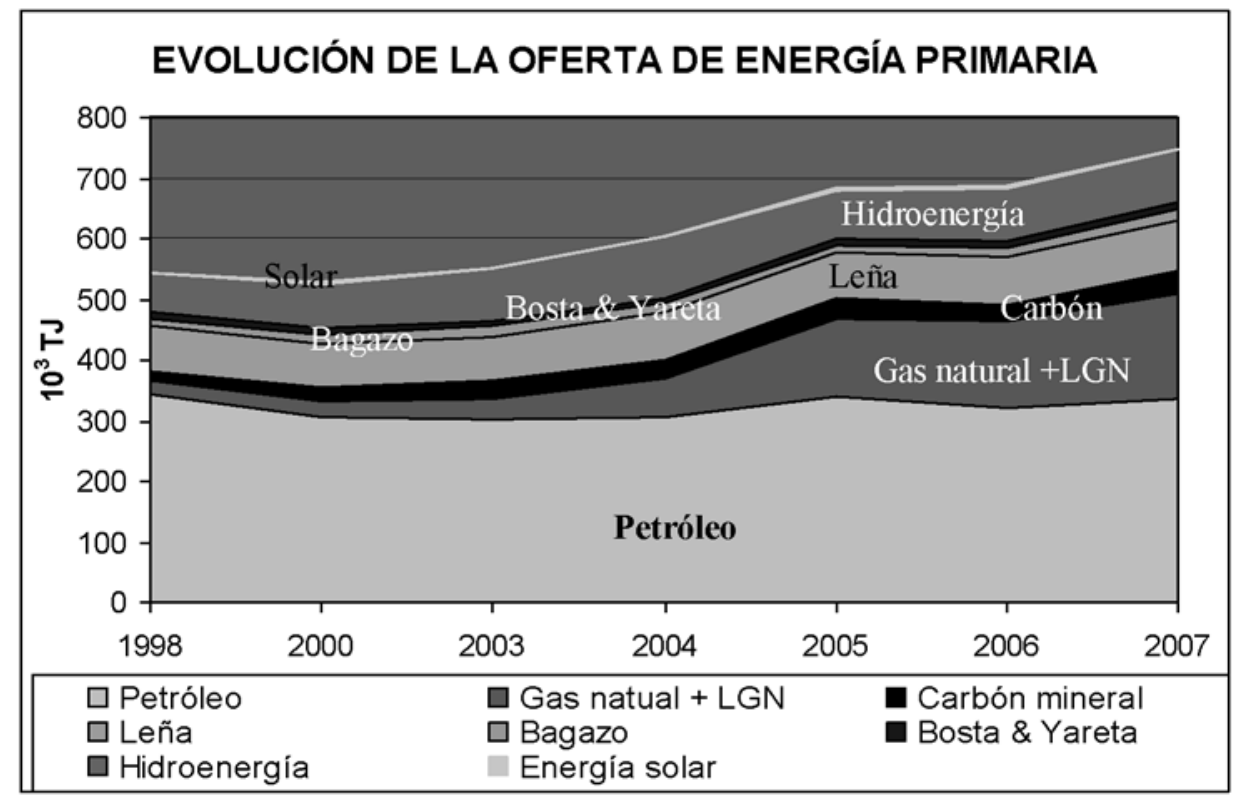

Fuente: Balances nacionales de energía (1998-2007) - MEM.

Para lograr resultados más exactos, es necesario ingresar información (consumo energético e indicadores energéticos) actualizada. La data nacional en el sector estatal no está actualizada, el último balance nacional de energía útil es del año 1998 y el de energía final es del año 2007; por ello, se deben realizar auditorías energéticas y encuestas por sectores y subsectores homogéneos para determinar con detalle valores de los principales indicadores energéticos, esto permitirá el uso de diversos modelos energéticos (de usos finales y/o mixtos) que como el MAED_D requieren de estos indicadores para realizar la evaluación de la demanda nacional de energía.

\section{EVOLUCIÓN DEL CONSUMO DE ENERGÍA EN EL PERÚ}

1.1. El consumo nacional de energía final del año 2007 por sectores económicos fue de 518982 TJ como se observa en la Figura 1, el sector que consumió más energía es el sector residencial, comercial y público con 169349 TJ con una participación del 32,6\%.

1.2. Evolución de la oferta de energía primaria y secundaria

La tendencia de la oferta de energía primaria y secundaria es de crecimiento desde el año 2000 , con un mayor incremento el año 2004 con el ingreso del proyecto Camisea. 
FIGURA 3. EVOLUCIÓN DE LA OFERTA DE ENERGÍA SECUNDARIA (1998-2007)

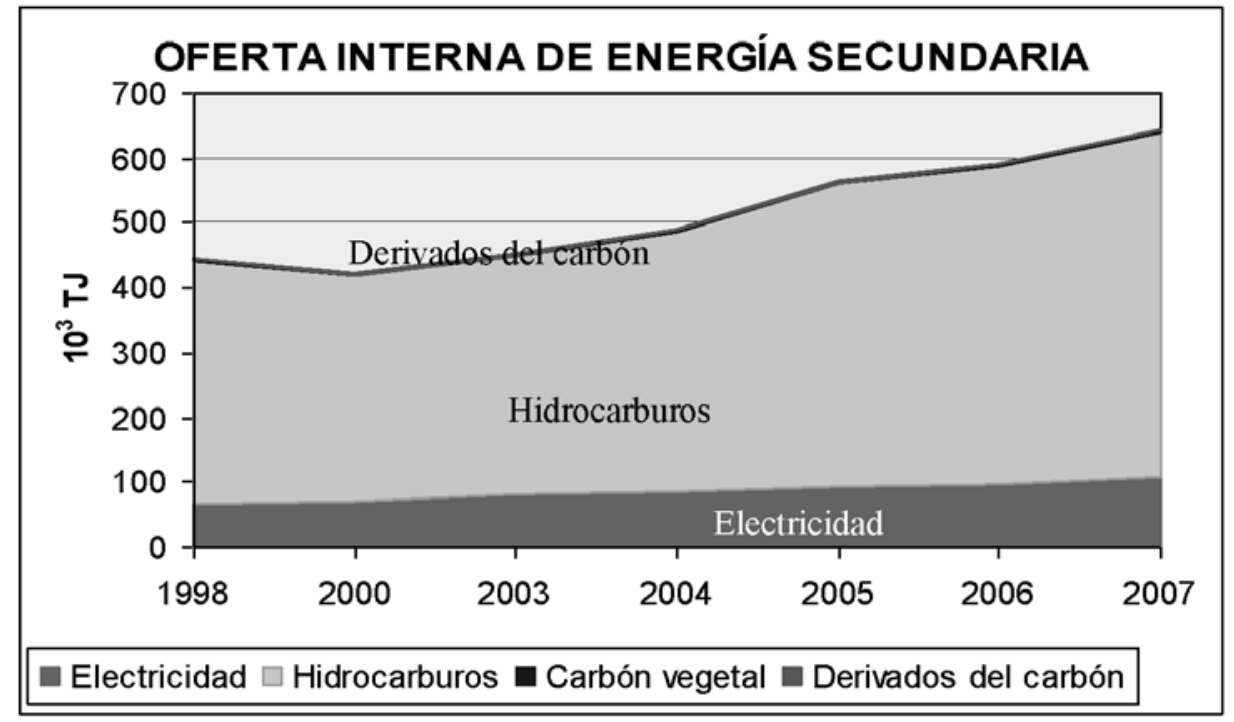

Fuente: Balances nacionales de energía (1998 - 2007) - MEM.

FIGURA 4. EVOLUCIÓN DE LA TASA DE CRECIMIENTO DE CONSUMO DE ENERGÍA ELÉCTRICA

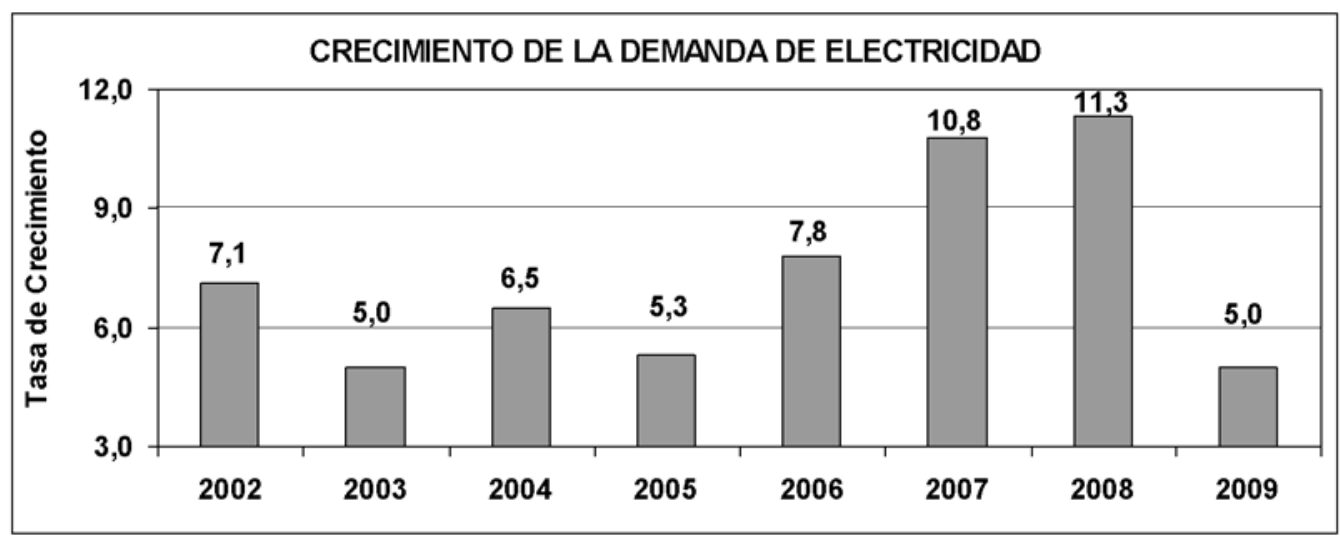

Fuente: MEM (DGE) - SEIN.

En el año 2007, la oferta interna de energía primaria fue de 746869 TJ como se observa en la Figura 2. La energía comercial conformada por: petróleo crudo, gas natural, líquidos de gas natural, carbón mineral y hidroenergía representó el $84,8 \%$ del total de la oferta interna bruta.

La producción de energía secundaria durante el año 2007 fue de 645493 TJ como se observa en la Figura 3 . En la estructura de consumo continúan predominando los hidrocarburos obtenidos de las refinerías y plantas de gas, que participan con el $82,4 \%$ del total producido.

\subsection{Evolución de la demanda de energía eléctrica} El crecimiento económico del país en especial durante los años 2006 al 2008 han generado el crecimiento del consumo de energía en diversos sectores, en el presente año (2009) a pesar de la crisis económica internacional la tasa de crecimiento del consumo eléctrico es del $5 \%$ como se muestra en la Figura 4.

1.4. Evolución de la máxima demanda y la reserva eléctrica

La máxima demanda de energía eléctrica registrada en el Sistema Interconectado Nacional tiene una tendencia ascendente, en el año 2003 la máxima demanda era inferior a los $3000 \mathrm{MW}$, el año 2009 esta cifra sobrepasa los $4100 \mathrm{MW}$ (Figura 5).

La Figura 6 muestra el mayor crecimiento de la oferta sobre la máxima demanda de energía eléctrica, lo que origina la disminución notable de la reserva eléctrica y por lo tanto se incrementa el riesgo de suministro del sistema eléctrico (cualquier desperfecto en alguna central de mediana magnitud afectaría el servicio eléctrico con cortes del suministro eléctrico). 
FIGURA 5. EVOLUCIÓN DE LA MÁXIMA DEMANDA DE ENERGÍA ELÉCTRICA DEL SEIN

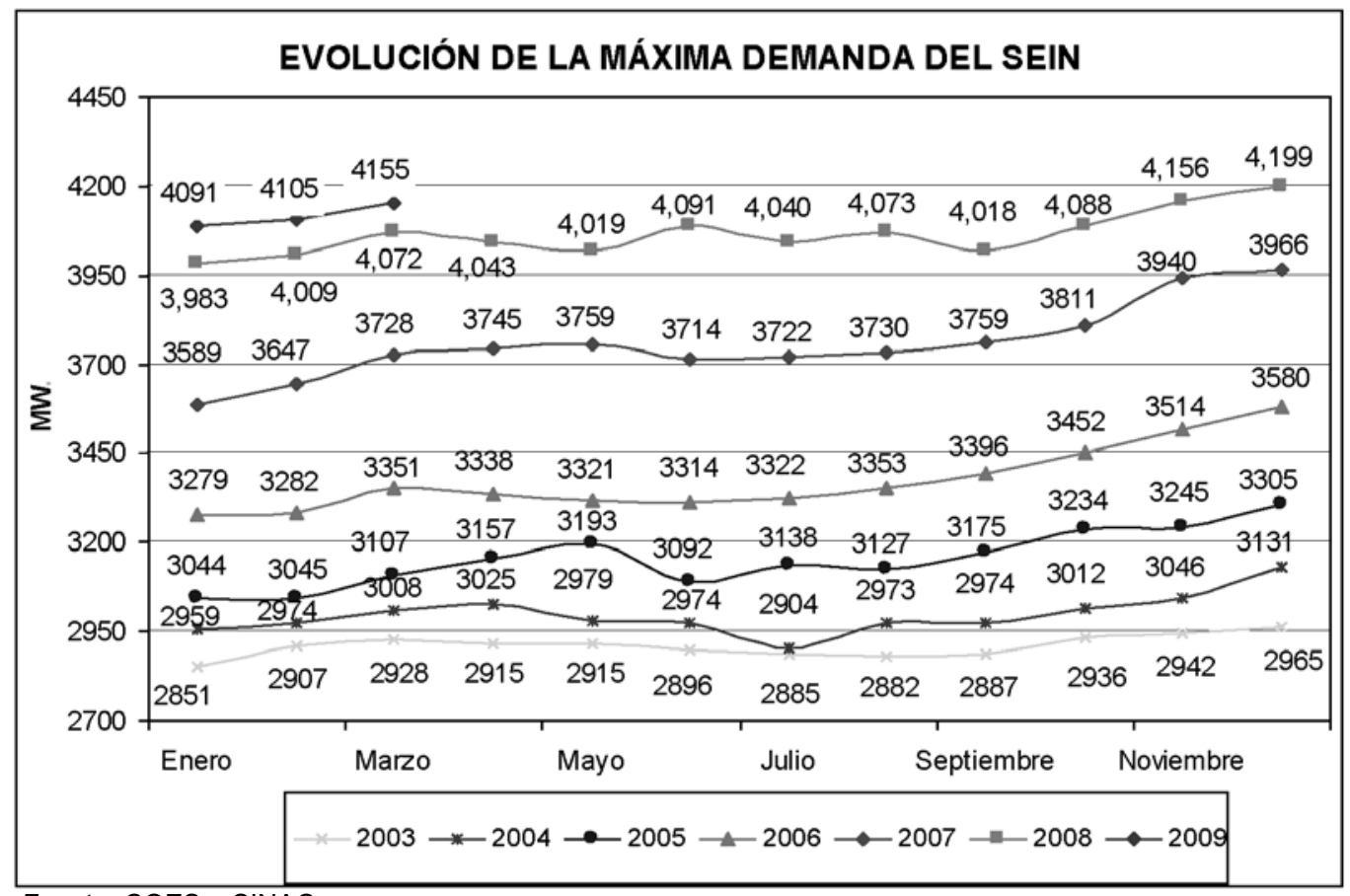

Fuente: COES - SINAC

FIGURA 6. MÁXIMA DEMANDA Y RESERVA ELÉCTRICA DEL SEIN (1998-2009)

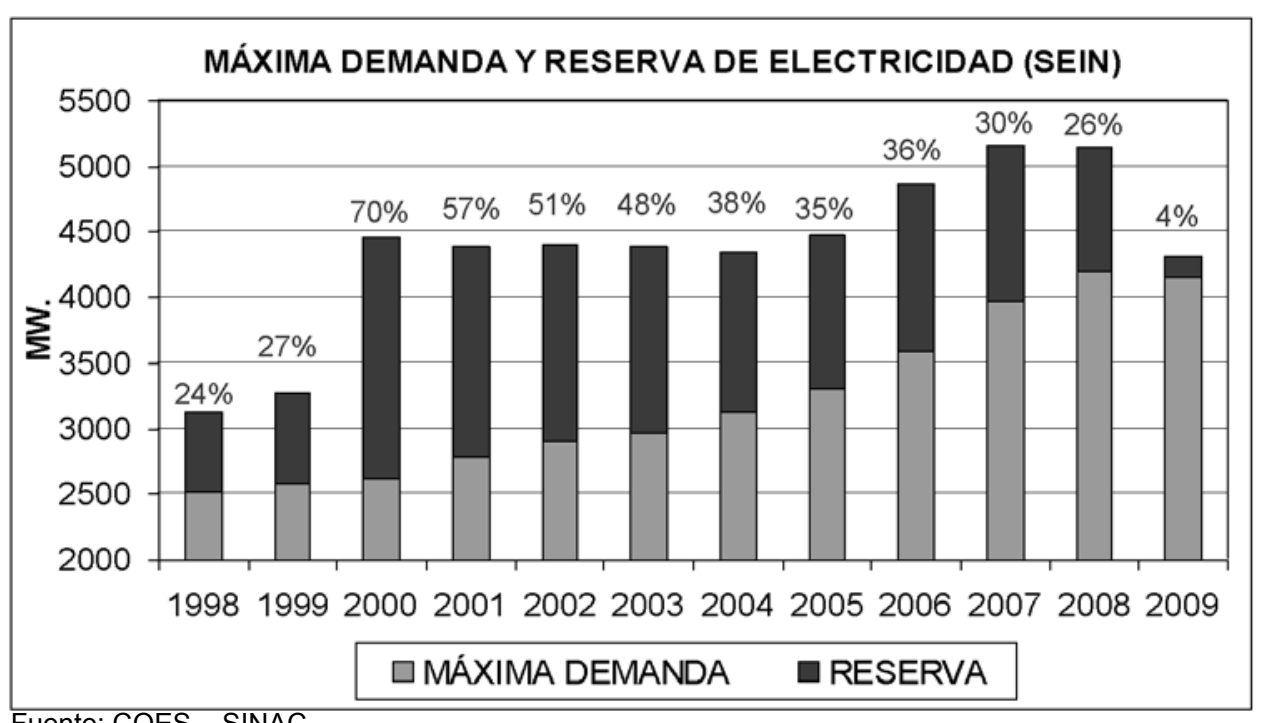

\section{CAUSAS Y EFECTOS DE LA CRISIS ENERGÉTICA EN EL PAÍS}

En la Figura 7, se muestran las causas y efectos de la crisis energética. Las causas están relacionadas con los recursos energéticos nacionales, la importación de petróleo y el planeamiento energético nacional. Los efectos están relacionados con la inestabilidad política, socioeconómica y tecnológica, el desarrollo no sostenido, la inseguridad de suministro y el descuido del medio ambiente.

\section{VARIABLES EXPLICATIVAS DEL CONSUMO DE ENERGÍA}

Las variables explicativas de la demanda nacional de energía se pueden agrupar en cinco factores: climatológicos, sociales, económicos, tecnológicos y los hábitos de consumo, como se aprecia en la Figura 8. 
FIGURA 7. CAUSAS Y EFECTOS DE LA CRISIS ENERGÉTICA EN EL PAÍS

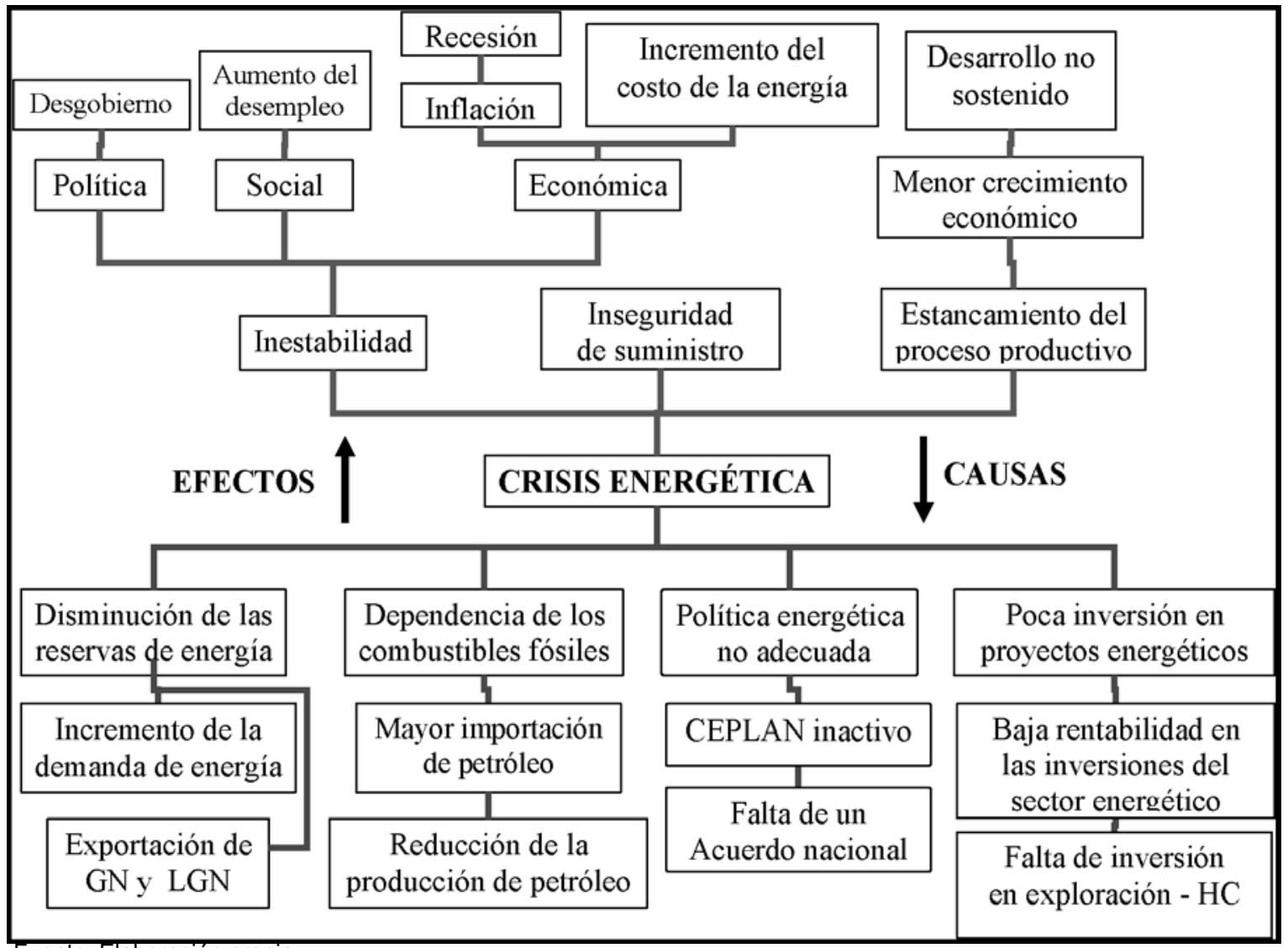

Fuente: Elaboración propia.

FIGURA 8. VARIABLES EXPLICATIVAS DEL CONSUMO DE ENERGÍA ELÉCTRICA

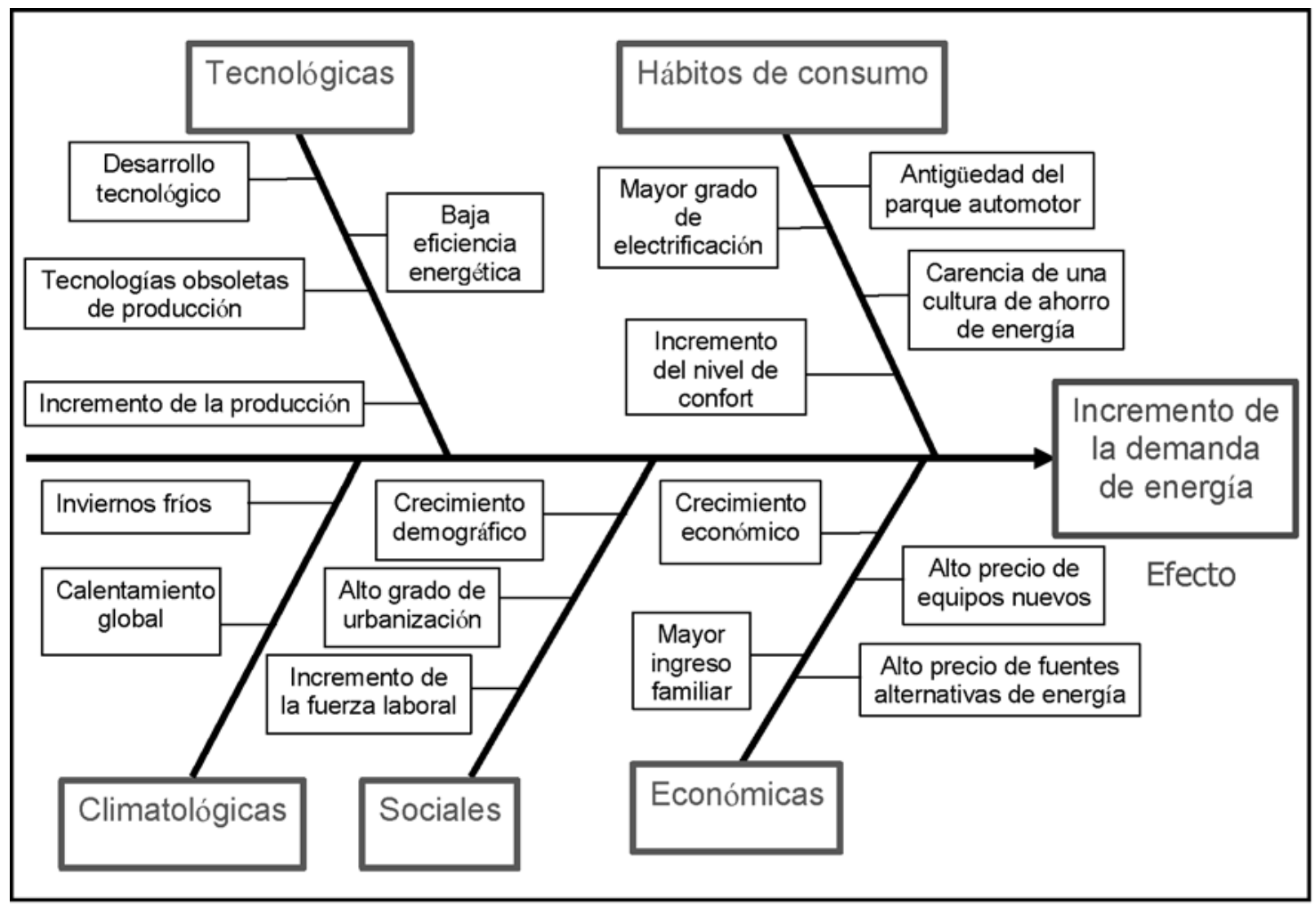

Fuente: Elaboración propia. 
TABLA 1. HOJAS DE CONTENIDO DEL MODELO MAED_D

\begin{tabular}{|c|c|c|c|}
\hline \multicolumn{2}{|r|}{ Tabla de contenido } & \multirow{2}{*}{$\begin{array}{c}\text { H. de datos } \\
\text { Notes }\end{array}$} & \multirow[t]{2}{*}{ No Tabla } \\
\hline 1 & Notas & & \\
\hline 2 & Descripción & Descr & \\
\hline 3 & Definiciones & Defs & \\
\hline 4 & Demografía & Demog-D & 1 \\
\hline 5 & Formación del PBI & GDP-D & 2 \\
\hline 6 & Intensidades energéticas & Enlnt-D & 3 \\
\hline 7 & Demanda de energía útil en el sector industria & UsEne-D & 4 \\
\hline 8 & Factores para los sectores agricultura, construcción y minería & ACMFac-D & 5 \\
\hline 9 & Demanda de energía final en la agricultura, construcción y minería & FIN_ACM & 6 \\
\hline 10 & Factores para la demanda de energía térmica útil en el sector manufacturero & ManFac1-D & 7 \\
\hline 11 & Factores para el sector manufacturero & ManFac2-D & 8 \\
\hline 12 & Demanda de energía final en la industria & FIN_Ind-D & 9 \\
\hline 13 & Transporte de carga & FrTrp-D & 10 \\
\hline 14 & Transporte de pasajeros en las ciudades & PassIntra-D & 11 \\
\hline 15 & Transporte de pasajeros entre las ciudades & PassInter-D & 12 \\
\hline 16 & Demanda de energía final en el sector transporte & FIN_Trp-D & 13 \\
\hline 17 & Demanda de energía útil en el sector residencial urbano & US_HH_Ur-D & 14 \\
\hline 18 & Demanda de energía útil en el sector residencial rural & US_HH_Rr-D & 15 \\
\hline 19 & Demanda de energía final en el sector residencial & FIN_HH-D & 16 \\
\hline 20 & Demanda de energía útil en el sector servicios & US_SS-D & 17 \\
\hline 21 & Factores para el sector servicios & SS_Fac-D & 18 \\
\hline 22 & Demanda de energía final en el sector servicios & FIN_SS-D & 19 \\
\hline 23 & Demanda total de energía final & Final-D & 20 \\
\hline 24 & Demanda total de energía final (definidas por el usuario) & Final_User-D & 21 \\
\hline 25 & Factores de conversión & Convs & \\
\hline
\end{tabular}

Fuente: Manual del Usuario MAED_D - OIEA, 2007.

\section{MODELO DE ANÁLISIS DE DEMANDA DE ENERGÍA (MAED)}

El software MAED es un modelo de simulación diseñada para reflejar los cambios estructurales en la demanda de energía de un país en el mediano y largo plazo. La demanda de energía se desagrega en un gran número de categorías de uso final que son calculadas mediante formulas del modelo. El nivel de la demanda de energía en el sector residencial está influenciado por diversas variables explicativas.

La metodología del MAED fue desarrollada originalmente por Messrs. B. Chateau y B. Lapillone (MEDEE: Modèle d'Evolution de la Demande d'Energie) del Institut Economique et Jurique de l'Energie (IEJE) de la Universidad de Grenoble, Francia (1980) y modificada por el Organismo Internacional de Energía Atómica (OIEA) quien después le denominó MAED_D.

EI MAED_D es utilizado en diversos países y organizaciones internacionales como: OIEA, AIE, OLA-
DE y CEPAL para la proyección del consumo nacional y regional de energía. El manual del software está disponible en Internet en: www-pub.iaea.org/ MTCD/publications/PDF/CMS-18_web.pdf

EI MAED_D se suministra en un libro de trabajo EXCEL y contiene 27 hojas, de las cuales 22 sirven como hojas de cálculo para ingresar datos y/o realizar cálculos del modelo, las otras 5 proporcionan información general como se muestra en la tabla 1 "TOC". Para el presente artículo sólo se utilizó 5 hojas de cálculo (Defs, Demog-D, US_HH_Ur-D, US_HH_Rr-D y FIN_HH-D) correspondientes al sector residencial.

El ingreso de datos se realiza mediante la hoja definiciones. Los datos como el número de años de proyección y el año base serán ingresados en las celdas B6 y B7 respectivamente. Para los tipos de vivienda en este artículo se utilizó las viviendas urbanas y rurales como se muestra en la Figura 9 , pero en el modelo se puede definir hasta 10 tipos diferentes de viviendas. 
FIGURA 9. CONSTRUCCIÓN DE LA ESTRUCTURA DEL MODELO MAED_D

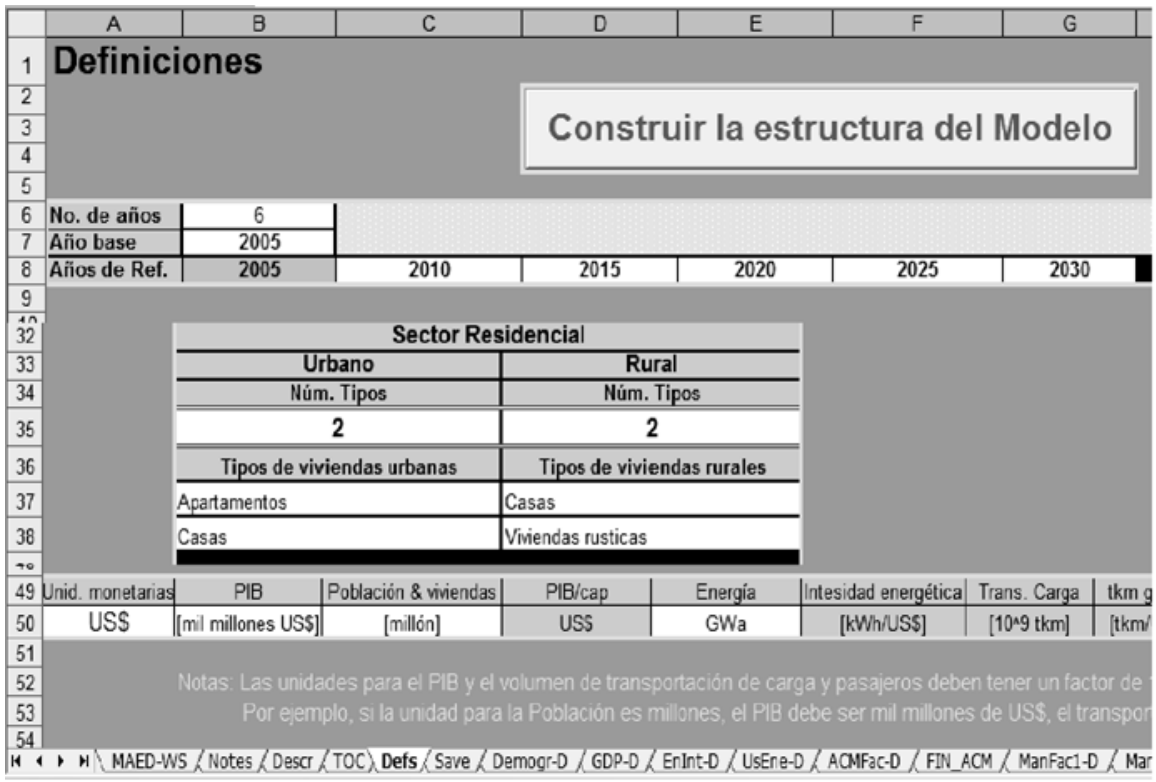

4.1. Cálculo del crecimiento demográfico: El crecimiento demográfico incrementa el número de residencias, lo que ocasiona el aumento del consumo energético. Para determinar la proyección poblacional se utilizó la ecuación 1.

$$
P_{n}=P_{0} \times(1+t)^{n}(\text { Ecuación 1) }
$$

Donde:

$P_{n}$ : tamaño de la población en el año " $n$ "

$\mathrm{P}_{0}$ : tamaño de la población actual

(Millones de personas)

$\mathrm{n}$ : número de años

(Millones de personas)

$\mathrm{t}$ : tasa de crecimiento anual de la población

(Años)

CUADRO 1: POBLACIÓN NACIONAL SEGÚN CENSOS (1940 - 2007)

\begin{tabular}{|c|c|c|c|}
\hline \multirow{2}{*}{ AÑO } & \multicolumn{3}{|c|}{ POBLACIÓN } \\
\cline { 2 - 4 } & CENSADA & OMITIDA & TOTAL \\
\hline 1940 & 6207967 & 815144 & 7023111 \\
\hline 1961 & 9906746 & 513611 & 10420357 \\
\hline 1972 & 13583208 & 583356 & 14166564 \\
\hline 1981 & 17005210 & 757021 & 17762231 \\
\hline 1993 & 22048356 & 591087 & 22639443 \\
\hline $2005 \mathrm{a} /$ & 26152265 & 1066999 & 27219264 \\
\hline 2007 & 27412157 & 808607 & 28220764 \\
\hline
\end{tabular}

a/ Censo de derecho o de jure. Información recopilada en el lugar de residencia.

Fuente: INEI. Censos Nacionales de Población y Vivienda, 1940-2007.
En el Cuadro 1, se muestran los censos poblacionales del Perú de los últimos 70 años, para la proyección de la población se tomó datos de los dos últimos censos, estos datos y otros relacionados con la demografía son ingresados al MAED en la hoja "Demogr-D".

En el Cuadro 2, se muestran los resultados de la proyección de la población para el periodo 2005 2030. Estos resultados son mostrados en el MAED aplicando la ecuación N. ${ }^{\circ} 1$ en la hoja "Demogr-D".

\section{USOS ENERGÉTICOS EN EL SECTOR RESIDENCIAL}

En el Cuadro 4, se muestra un resumen por categoría de uso final y formas de energía disponibles en el sector residencial que son: calefacción, calentamiento de agua, aire acondicionado y equipos domésticos secundarios (refrigeradoras, luminarias, lavadoras, etc.). Estos datos servirán para definir la estructura del modelo.

En el Cuadro 5 se muestra un resumen de los datos básicos para la demanda de energía útil.

En el Cuadro 6 se muestra un resumen de los requerimientos energéticos básicos del sector residencial.

Estos datos de los Cuadros 5 y 6 son ingresados en el MAED en las Tablas 14 y 15 en las hojas "US_HH_Ur-D" y "US_HH_Rr-D" respectivamente. En esta misma hoja se calculan dichos resultados. 
CUADRO 2. PROYECCIÓN DE LOS FACTORES DEMOGRÁFICOS (2005-2030)

\begin{tabular}{|l|c|c|c|c|c|c|c|}
\hline \multicolumn{1}{|c|}{ ITEM } & Unidad & 2005 & 2010 & 2015 & 2020 & 2025 & 2030 \\
\hline Población & [millón] & $\mathbf{2 7 . 2 1 9}$ & $\mathbf{2 8 . 4 2 4}$ & $\mathbf{2 9 . 7 8 8}$ & $\mathbf{3 1 . 2 1 8}$ & $\mathbf{3 2 . 7 1 6}$ & $\mathbf{3 4 . 2 8 7}$ \\
\hline Tasa de crecimiento & [\% p. a.] & n. a. ${ }^{* *}$ & 0.870 & 0.942 & 0.942 & 0.942 & 0.942 \\
\hline Población Urbana & [\%] & $\mathbf{7 4 . 2 9 4}$ & $\mathbf{7 5 . 1 6 5}$ & $\mathbf{7 6 . 4 5 2}$ & $\mathbf{7 7 . 8 4 0}$ & $\mathbf{7 9 . 0 2 3}$ & $\mathbf{7 9 . 0 2 3}$ \\
\hline Habitantes/casa & [cap] & 3.700 & 3.571 & 3.446 & 3.325 & 3.209 & 3.096 \\
\hline N. ${ }^{\circ}$ de Viviendas & [millón] & $\mathbf{5 . 4 6 5}$ & $\mathbf{5 . 9 8 4}$ & $\mathbf{6 . 6 1 0}$ & $\mathbf{7 . 3 0 8}$ & $\mathbf{8 . 0 5 8}$ & $\mathbf{8 . 7 5 1}$ \\
\hline Población Rural & [\%] & $\mathbf{2 5 . 7 0 6}$ & $\mathbf{2 4 . 8 3 5}$ & $\mathbf{2 3 . 5 4 8}$ & $\mathbf{2 2 . 1 6 0}$ & $\mathbf{2 0 . 9 7 7}$ & $\mathbf{2 0 . 9 7 7}$ \\
\hline Habitantes/casa & [cap] & 4.200 & 4.074 & 3.952 & 3.833 & 3.718 & 3.607 \\
\hline N. . de Viviendas $^{\text {[millón] }}$ & $\mathbf{1 . 6 6 6}$ & $\mathbf{1 . 7 3 3}$ & $\mathbf{1 . 7 7 5}$ & $\mathbf{1 . 8 0 5}$ & $\mathbf{1 . 8 4 6}$ & $\mathbf{1 . 9 9 4}$ \\
\hline F. laboral potencial & [\%] & 57.240 & 58.957 & 61.905 & 65.000 & 68.250 & 71.663 \\
\hline F. laboral trabajando & [\%] & 80.160 & 81.763 & 83.398 & 85.066 & 86.768 & 88.503 \\
\hline F. laboral activa & [millón] & $\mathbf{1 2 . 4 8 9}$ & $\mathbf{1 3 . 7 0 2}$ & $\mathbf{1 5 . 3 7 9}$ & $\mathbf{1 7 . 2 6 1}$ & $\mathbf{1 9 . 3 7 4}$ & $\mathbf{2 1 . 7 4 6}$ \\
\hline $\begin{array}{l}\text { Población en } \\
\text { grandes ciudades }\end{array}$ & [millón] & $\mathbf{1 2 . 2 4 9}$ & $\mathbf{1 2 . 9 8 2}$ & $\mathbf{1 3 . 8 1 0}$ & $\mathbf{1 4 . 6 9 0}$ & $\mathbf{1 5} \mathbf{. 6 2 6}$ & $\mathbf{1 6 . 6 2 1}$ \\
\hline
\end{tabular}

n. a. ** (no aplicable).

Fuente: Perfil sociodemográfico del Perú SEIN - INEI (Lima, agosto 2008).

CUADRO 4. CATEGORÍA DE USO FINAL DE ENERGÍA EN EL SECTOR RESIDENCIAL

\begin{tabular}{|l|c|c|c|c|c|}
\hline \multirow{2}{*}{ Formas de energía } & \multicolumn{5}{|c|}{ Categoría de uso final } \\
\cline { 2 - 6 } & Calefacción & $\begin{array}{c}\text { Calentamiento } \\
\text { de agua }\end{array}$ & Cocción & Aire \\
acondicionado & Electrodomésticos \\
\hline Combustibles radicionales & $\mathrm{X}$ & $\mathrm{X}$ & $\mathrm{X}$ & & $\mathrm{X}$ \\
\hline Electricidad & $\mathrm{X}$ & $\mathrm{X}$ & $\mathrm{X}$ & $\mathrm{X}$ & \\
\hline Solar térmica & $\mathrm{X}$ & $\mathrm{X}$ & $\mathrm{X}$ & & $\mathrm{X}$ \\
\hline Combustibles fósiles & $\mathrm{X}$ & $\mathrm{X}$ & $\mathrm{X}$ & $\mathrm{X}$ & \\
\hline
\end{tabular}

Fuente: BNE - MEM, MAED_D estructura del modelo.

\section{CUADRO 5. FACTORES DE VIVIENDA PARA LOS USOS DEL SECTOR RESIDENCIAL}

\begin{tabular}{|l|r|r|r|}
\hline \multicolumn{1}{|c|}{ Ítem } & Unidad & \multicolumn{1}{c|}{ Urbano } & \multicolumn{1}{c|}{ Rural } \\
\hline Viviendas & {$[$ millón] } & 5.465 & 1.666 \\
\hline Porcentaje de apartamentos/viviendas rústicas & {$[\%]$} & 11.000 & 20.000 \\
\hline Porcentajes de casas & {$[\%]$} & 89.000 & 80.000 \\
\hline Tamaño de la vivienda: apartamento/vivienda rústica & {$[\mathrm{m} 2]$} & 90.000 & 40.000 \\
\hline Tamaño de la vivienda casas & {$[\mathrm{m} 2]$} & 120.000 & 60.000 \\
\hline Vivienda con aire acondicionado - apartamentos & {$[\%]$} & 15.000 & 0 \\
\hline Vivienda con aire acondicionado - casas & {$[\%]$} & 12.500 & 0 \\
\hline Coeficiente de electrificación de las viviendas/sector & {$[\%]$} & 95.000 & 65.000 \\
\hline Penetración de los Comb. tradicionales en la cocción & {$[\%]$} & 19.752 & 90.419 \\
\hline Penetración de la electricidad en cocción & {$[\%]$} & 9.977 & 0.742 \\
\hline Penetración de la energía solar térmica en cocción & {$[\%]$} & 7.180 & 1.121 \\
\hline Penetración de los combustibles fósiles en cocción & {$[\%]$} & 63.100 & 7.700 \\
\hline Eficiencia de los combustibles tradicionales & {$[\%]$} & 13.549 & 14.680 \\
\hline Eficiencia de los combustible fósiles & {$[\%]$} & 53.389 & 52.286 \\
\hline Participación de la solar térmica & {$[\%]$} & 100.000 & 100.000 \\
\hline
\end{tabular}

Fuente: INEI, Estudio Integral de Energía.

CUADRO 6. REQUERIMIENTOS DEL SECTOR RESIDENCIAL

\begin{tabular}{|l|c|r|r|}
\hline \multicolumn{1}{|c|}{ Ítem } & Unidad & Urbano & Rural \\
\hline $\begin{array}{l}\text { Requerimiento esp. AC- } \\
\text { Apartamentos }\end{array}$ & {$[\mathrm{kW} . \mathrm{h} /$ vivienda/año] } & 768.000 & 0 \\
\hline Requerimiento esp. AC-Casas & {$[\mathrm{kW} . \mathrm{h} /$ vivienda/año] } & 1536.000 & 0 \\
\hline $\begin{array}{l}\text { Requerimientos de energía para } \\
\text { cocción }\end{array}$ & {$[\mathrm{kW} \cdot \mathrm{h} /$ vivienda/año] } & 1170.592 & 1602.704 \\
\hline $\begin{array}{l}\text { Consumo de electricidad en } \\
\text { equipamiento }\end{array}$ & {$[\mathrm{kW} . \mathrm{h} /$ vivienda/año] } & 806.548 & 112.644 \\
\hline $\begin{array}{l}\text { Combustibles fósiles para la } \\
\text { iluminación }\end{array}$ & {$[\mathrm{kW} . \mathrm{h} /$ vivienda/año] } & 180.581 & 176.742 \\
\hline
\end{tabular}

Fuente: INEI, Estudio Integral de Energía. 
FIGURA 10. DEMANDA DE ENERGÍA FINAL DEL SECTOR RESIDENCIAL URBANO

\begin{tabular}{|c|c|c|c|c|c|c|}
\hline $\begin{array}{r}\text { De } \\
\text { Residen }\end{array}$ & $\begin{array}{l}\text { nand } \\
\text { ial U }\end{array}$ & $\begin{array}{l}\text { de E } \\
\text { ano }\end{array}$ & $\begin{array}{l}\text { rgía F } \\
\text { scena }\end{array}$ & $\begin{array}{l}\text { nal se } \\
\text { o de }\end{array}$ & $\begin{array}{l}\text { tor } \\
\text { eferer }\end{array}$ & \\
\hline 5.4 & & & & Solar & érmica & \\
\hline 3.6 & & & & & & \\
\hline 2.7 & & & & C. Fó & & \\
\hline 1.8 & & & Elec & cidad & & \\
\hline 0.9 & & & & & & \\
\hline 0.0 & & & ombust1 & les Irac & ionales & \\
\hline & 2005 & 2010 & 2015 & 2020 & 2025 & 2030 \\
\hline - Solar térmica & 0.052 & 0.057 & 0.067 & 0.077 & 0.089 & 0.102 \\
\hline $\begin{array}{l}\square \text { Combustibles } \\
\text { fósiles }\end{array}$ & 0.869 & 0.950 & 1.101 & 1.277 & 1.478 & 1.683 \\
\hline$\square$ Electricidad & 0.597 & 0.661 & 0.770 & 0.899 & 1.046 & 1.203 \\
\hline $\begin{array}{c}\text { Combustibles } \\
\text { tradicionales }\end{array}$ & 1.065 & 1.166 & 1.352 & 1.570 & 1.817 & 2.072 \\
\hline
\end{tabular}

FIGURA 11. DEMANDA DE ENERGÍA FINAL DEL SECTOR RESIDENCIAL RURAL

\begin{tabular}{|c|c|c|c|c|c|c|}
\hline $\begin{array}{r}\text { Demanda } \\
\mathbf{R}\end{array}$ & $\begin{array}{l}\text { de E } \\
\text { Iral E }\end{array}$ & $\begin{array}{l}\text { rgía } \\
\text { enar }\end{array}$ & $\begin{array}{l}\text { el } S \\
\text { de } R\end{array}$ & $\begin{array}{l}\text { tor } R \\
\text { feren }\end{array}$ & $\begin{array}{l}\text { siden } \\
\mathbf{a}\end{array}$ & \\
\hline 2.8 & & ectricid & & E. & lar & \\
\hline 0.7 & & & nbustib & s Tradi & nales & \\
\hline 0.0 & 2005 & 2010 & 2015 & 2020 & 2025 & 2030 \\
\hline Golar térmica & 0.003 & 0.005 & 0.008 & 0.012 & 0.018 & 0.025 \\
\hline$\square$ Electricidad & 0.016 & 0.022 & 0.032 & 0.051 & 0.072 & 0.106 \\
\hline$\square$ Combustibles fósiles & 0.057 & 0.075 & 0.086 & 0.086 & 0.095 & 0.129 \\
\hline $\begin{array}{c}\square \text { Combustibles } \\
\text { tradicionales }\end{array}$ & 1.877 & 1.877 & 2.028 & 2.172 & 2.309 & 2.524 \\
\hline
\end{tabular}

\section{RESULTADOS Y SU DISCUSIÓN}

En esta sección se muestra el comportamiento de la demanda de energía final simulado en el MAED_D para el sector residencial urbano, rural y la suma de ambos subsectores en el periodo de proyección (2005-2030) por tipo de combustibles para el único escenario de referencia modelado.

En la Figura 10 se muestra el comportamiento del consumo proyectado para el sector residencial urbano, se observa que el consumo de los combustibles tradicionales crecerá desde $1.065 \mathrm{GWa}$ hasta $2.072 \mathrm{GWa}$.

En la Figura 11 se muestra el comportamiento del consumo proyectado de energía final para el sector residencial rural, se observa que el consumo de combustibles tradicionales se incrementará desde $1.877 \mathrm{GWa}$ en el año 2005 hasta 2.524 GWa en el año 2030.
En la Figura 12 se muestra la suma de los consumos del sector residencial urbano y rural por tipos de combustibles, se observa que los combustibles tradicionales se incrementarán desde $2.942 \mathrm{GWa}$ hasta $4.596 \mathrm{GWa}$.

\section{CONCLUSIONES}

En el sector residencial al aplicar la metodología MAED_D, considerando una tasa de crecimiento demográfico anual de $0.942 \%$ proyecta que la demanda nacional de energía final se incremente desde 4.536 GWa en el 2005 hasta 7.845 GWa en el 2030.

Es un modelo adecuado para determinar la proyección del consumo nacional de energía en el mediano y largo plazo porque es un modelo de usos finales, por esta razón es usado en diversos organismos internacionales (OIEA, OLADE y CEPAL). 
FIGURA 12 DEMANDA DE ENERGÍA FINAL DEL SECTOR RESIDENCIAL

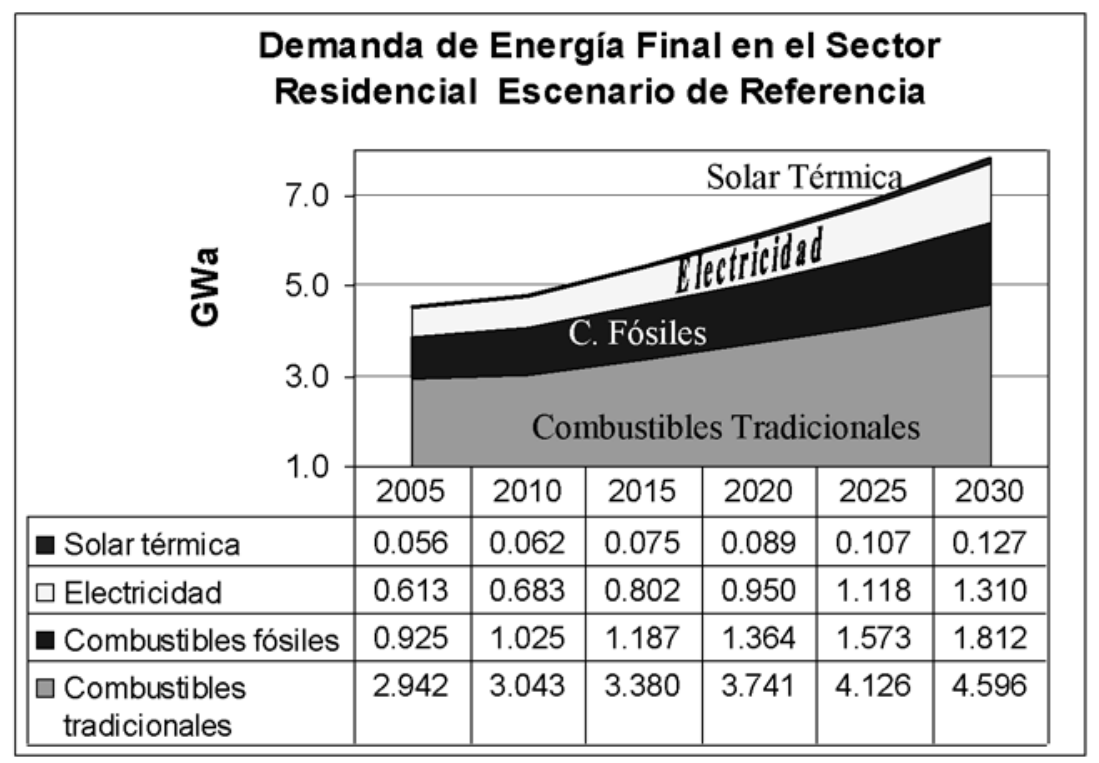

De mantenerse el ritmo de crecimiento de la demanda nacional de energía alrededor del $5 \%$ anual, sin el incremento de la oferta mayor al $2 \%$ anual y sin el incremento de las reservas de energía especialmente de gas natural y petróleo. Aproximadamente a partir del año 2012 se agravará la crisis energética.

\section{RECOMENDACIONES}

Implementar a nivel nacional una cultura de ahorro y uso eficiente de la energía, esto permitirá reducir los niveles de consumo total de energía hasta en un $40 \%$ (OLADE), teniendo en cuenta que en el Perú sólo el $37 \%$ de la energía primaria se convierte en energía útil.

No es recomendable analizar la demanda nacional de energía utilizando variables correlativas, porque sería necesario disponer de información detallada del sector energético y se pueden duplicar u omitir algunos segmentos de las cadenas energéticas de las fuentes de energía comercial y no comercial, esta última con mayor incidencia porque al no ser comerciales no se lleva un control estricto de su consumo.

Incentivar y apoyar el desarrollo sostenible con fuentes renovables y/o más eficientes en especial en las zonas rurales donde los costos de los combustibles debido al transporte son más elevados. Ampliar el horizonte energético mediante el incentivo a la exploración petrolera y gasífera para incrementar las reservas con participación del Estado y la empresa privada nacional y extranjera.
Impulsar la investigación de energías renovables y hacer estudios y auditorías energéticas en los diferentes sectores energéticos a nivel nacional con el fin de actualizar los distintos indicadores energéticos para que los estudios de proyección se ajusten más a la realidad del sector energético y se logre un mejor planeamiento energético integral de energía.

\section{REFERENCIAS BIBLIOGRÁFICAS}

Bouille, D.H. (2004). Economía de la energía, Instituto de la energía, Fundación Bariloche, Argentina.

INEI Compendio estadístico 2005-2007: escrito y publicado por el Sistema Estadístico Nacional (SEN) Lima.

INEI-UNFPA-PUND. Perfil sociodemográfico del Perú: censos 2007 de población y vivienda; agosto.

OIEA-AIE-EUROSTAT-AEMA (2008). Indicadores energéticos para el desarrollo sostenible; directrices y metodologías; Austria.

OLADE (1998). Energía y desarrollo sustentable en América Latina y el Caribe: Enfoques para la política energética. Quito Ecuador. CEPAL, GTZ Editores.

Organismo Internacional de Energía Atómica (2007). Modelo para el análisis de la demanda de energía (MAED_D). Manual del Usuario, Viena. 


\section{BIBLIOGRAFÍA EXTRAÍDA DE INTERNET}

MEM, Balance de Energía Útil: 1998. Escrito y publicado por la OGPP el año 2000 [Internet], [acceso 12 noviembre 2007] disponible en: http:// www.minem.gob.pe/hidrocarburos/pub_ogp_ balanenerutil1998.asp

MEM, Balance Nacional de energía 2005: Escrito y publicado por la Oficina de Planeamiento y Política Sectorial (OPPS) agosto del 2007 [acceso 12 noviembre 2007], disponible en: www.minem.gob.pe/archivos/dgh/publicaciones/oterg/ balance2005.pdf

MEM, Plan Referencial de energía al 2015: publicado por la Oficina Técnica de Energía (OTERG)
[Internet], [acceso 19 diciembre 2009], disponible en: www.minem.gob.pe/archivos/dgh/publicaciones/oterg.pdf

MEM, Plan referencial de hidrocarburos 20072016: Escrito y publicado por la Dirección General de Hidrocarburos 2006 (DGH) diciembre 2007, [acceso 03 noviembre 2009] disponible en: http://www.minem.gob.pe/hidrocarburos/ pub_planreferen_2006.asp

Utilización de indicadores energéticos en el planeamiento energético integrado. Río de Janeiro, febrero 2006. [acceso 28 de agosto 2009] disponible en: http://www.ppe.ufrj.br/ppe/production/ tesis/fmcima.pdf 